\title{
Malófagos (Phthiraptera) recolhidos de aves silvestres no Zoológico de São Paulo, SP, Brasil
}

\author{
Michel P. Valim ${ }^{1,2}$, Rodrigo H. F. Teixeira ${ }^{3}$, Marinete Amorim² $^{2}$ \& Nicolau M. Serra-Freire ${ }^{2}$
}

${ }^{1}$ Mestrando do Programa de Pós-graduação em Parasitologia-ICB/UFMG. Av. Presidente Antônio Carlos, 6627, $31270-901$ Belo Horizonte-MG, Brasil. mpvalim@hotmail.com.

${ }^{2}$ Laboratório de Ixodides, Departamento de Entomologia-Instituto Oswaldo Cruz/FIOCRUZ. Av. Brasil, 4365, $21045-900$ Rio de Janeiro-RJ, Brasil.

${ }^{3}$ Médico Veterinário do Zoológico de São Paulo. Av. Miguel Stefano, 4241, 04301-905 São Paulo-SP, Brasil.

\begin{abstract}
Chewing lice (Phthiraptera) from wild birds in the São Paulo Zoo, State of São Paulo, SP, Brazil. Twentyeight species of chewing lice were recovered from wild birds in the São Paulo Zoo during 2003. Twenty-three were identified to species level and five to the generic level. New species records for Brazil and new host-lice associations are discussed.
\end{abstract}

KEYWORDS. Amblycera, captive birds, host-lice relationships, Ischnocera, lice.

RESUMO. Malófagos (Phthiraptera) recolhidos de aves silvestres no Zoológico de São Paulo, SP, Brasil. Vinte e oito espécies de malófagos foram recolhidas de aves silvestres no acervo do Zoológico de São Paulo durante o ano de 2003. Vinte e três foram identificadas ao nível de espécie e cinco ao nível genérico. Os relatos de novas espécies no Brasil e novas associações parasitárias para a literatura são discutidos.

PALAVRAS-CHAVE. Amblycera, aves de cativeiro, Ischnocera, piolhos, relação parasito-hospedeiro.

Ainda que um relativo número de ectoparasitos possa ser encontrado sobre as aves, pouco se sabe em relação às doenças por eles causadas, o que em parte explica o pequeno número de estudos relacionados com o tema. Juntamente com os ácaros plumícolas (Acari: Acaridida), os malófagos são os parasitos mais freqüentemente encontrados sobre as aves silvestres. No Brasil poucos trabalhos tem dado ênfase a esses parasitos de aves cativas (Freitas et al. 2002; Silva et al. 2004).

A Ordem Phthiraptera é dividida em quatro subordens, das quais apenas duas (Ischnocera e Amblycera) são encontradas nas aves. Os Ischnocera vivem na plumagem ou na pele de seus hospedeiros e exibem alto grau de especificidade. A grande diversidade de hospedadores aliada às adaptações necessárias para habitar diferentes nichos ecológicos resultou em grande diversidade de formas, com mais de 3.000 espécies atualmente conhecidas. São insetos altamente especializados para viver sobre seus hospedeiros e a alta especificidade não é vista na maioria dos outros insetos ectoparasitos (Barker 1994; Smith 2001; Johnson \& Clayton 2003). Por passarem todo seu ciclo de vida sobre um único hospedeiro, na maioria dos casos cada espécie de malófago é restrita a uma espécie ou a um grupo próximo de hospedeiros. Esta distribuição sugere que eles se tornaram parasitos das aves em um estágio primitivo na evolução destes hospedeiros (Clay 1950), tornando possível o simples exame de um malófago um fator decisivo para determinar, muitas vezes, a ordem do respectivo hospedeiro.

O presente trabalho foi desenvolvido a partir de amostras obtidas no ano de 2003 no Zoológico de São Paulo e enviadas ao Laboratório de Ixodides, Departamento de Entomologia,
IOC/FIOCRUZ, tendo o cuidado de reconhecer equívocos na obtenção do material, denominados na literatura especializada como contaminação (Hopkins 1939; Pilgrim \& Palma 1982), tendo em vista a procedência de nossas amostras de determinado Zoológico. Dadas as limitações para captura de ectoparasitos permanentes em animais vivos confinados em zoológicos, e a escassez de dados conhecidos, o presente estudo adiciona informações à fauna de malófagos das aves do Brasil.

\section{MATERIAL EMÉTODOS}

Os ectoparasitos foram coletados durante o ano de 2003 sobre aves silvestres confinadas no Zoológico de São Paulo, SP. Após fixados em álcool etílico $70^{\circ} \mathrm{GL}$ foram enviados ao Laboratório de Ixodides, Fiocruz/RJ. Os piolhos foram montados em preparações permanentes segundo a técnica de rotina preconizada em Palma (1978).

A nomenclatura dos malófagos, bem como as associações da relação parasito/hospedeiro estão conforme Price et al. (2003) e a nomenclatura vulgar e específica das aves segue a publicação de Sick (1997).

\section{RESULTADOS}

A partir de amostras de animais atendidos no Departamento de Medicina Veterinária do Zoológico de São Paulo no ano de 2003 foi possível obter espécimes de malófagos de 23 espécies de aves, distribuídas por nove ordens e 14 famílias, assim nomeados em ordem alfabética: Anseriformes: Anatidae: 
Cygnus melanocoryphus (Molina, 1782), Dendrocygna bicolor (Vieillot, 1816); Ciconiiformes: Cathartidae: Coragyps atratus (Bechstein, 1793), Vultur gryphus Linnaeus, 1758; Ciconiidae: Jabiru mycteria (Lichtenstein, 1819); Phoenicopteridae: Phoenicopterus chilensis Molina, 1782; Falconiformes: Accipitridae: Leucopternis lacernulatus (Temminck, 1827), Rupornis magnirostris (Gmelin, 1788), Leucopternis polionota (Kaup, 1847), Elanus leucurus (Vieillot, 1818); Falconidae: Polyborus plancus (Miller, 1777); Galliformes: Cracidae: Penelope superciliaris Temminck, 1815, Crax fasciolata Spix, 1825, Aburria jacutinga (Spix, 1825); Passeriformes: Corvidae: Cyanocorax cristatellus (Temminck, 1823); Cotingidae: Procnias nudicollis (Vieillot, 1817); Turdidae: Turdus rufiventris Vieillot, 1818; Pelecaniformes: Fregatidae: Fregata magnificens Mathews, 1914; Psittaciformes: Psittacidae: Ara chloroptera Gray, 1859, Anodorhynchus hyacinthinus (Latham, 1790); Strigiformes: Strigidae: Pulsatrix perspicillata (Latham, 1790), Rhinoptynx clamator (Vieillot, 1807); Struthioniformes: Struthionidae: Struthio camelus Linnaus, 1758.

As seguintes espécies de malófagos, em ordem alfabética, foram reconhecidas: Acidoproctus rostratus (Rudow, 1866); Amyrsidea (Cracimenopon) purpurascens Carriker, 1950; Ardeicola leucosoma Kumar \& Tandan, 1968; Brueelia addoloratoi Cicchino, 1986; Brueelia nitzschi Kéler, 1938; Colpocephalum heterosoma Piaget, 1880; Colpocephalum maculatum Piaget, 1880; Colpocephalum mycteriae Price \& Beer, 1965; Colpocephalum napiforme Rudow, 1869; Colpocephalum trichosum Harrison, 1916; Cotingacola dimorpha Carriker, 1956; Craspedorrhynchus brevicapitis Carriker, 1956; Craspedorrhynchus sp.; Degeeriella elani Tendeiro, 1955; Degeeriella sp.; Holomenopon brevithoracicum (Piaget, 1880); Holomenopon leucoxanthum (Burmeister, 1838); Kurodaia (Conciella) crassiceps (Piaget, 1885); Labicotes guttatus (Taschenberg, 1882); Laemobothrion (Laemobothrion) glutinans Nitzsch [in Giebel], 1861; Menacanthus chaparensis Carriker, 1946; Myrsidea sp.; Oxylipeurus sp.; Pectinopygus fregatiphagus (Eichler, 1943); Psittacobrosus burmeisteri (Kellogg, 1906); Psittacobrosus sp.; Strigiphilus heterurus Carriker, 1966; Struthiolipeurus struthionis (Gervais, 1844).

Após identificação os dados foram agrupados por subordem, família e nome científico conforme Tabela I.

\section{DISCUSSÃO}

Das 28 espécies de malófagos encontradas 19 são pela primeira vez reportadas para o Brasil (Amyrsidea purpurascens; Colpocephalum heterosoma; C. trichosum; C. mycteriae; Holomenopon brevithoracicum; H. leucoxanthum; Kurodaia crassiceps; Menacanthus chaparensis; Psittacobrosus burmeisteri; Acidoproctus rostratus; Cotingacola dimorpha; Craspedorrhynchus brevicapitis; Labicotes guttatus; Pectinopygus fregatiphagus e Strigiphilus heterurus); quatro não determinadas (Myrsidea sp.; Psittacobrosus sp.; Degeeriella sp. e Oxylipeurus sp.)
Assunto controverso no estudo dos Phthiraptera, é a proposição de novos hospedeiros para espécies já descritas, dada a grande especificidade deste grupo de parasitos, mesmo assim consideramos ter encontrado sete novas associações na relação parasito-hospedeiro (Amyrsidea purpurascens / Penelope superciliaris; Colpocephalum mycteriae / Jabiru mycteria; Colpocephalum napiforme / Leucopternis lacernulata; Menacanthus chaparensis / P. superciliaris; Brueelia nitzschi / Cyanocorax cristatellus; Cotingacola dimorpha / Procnias nudicollis; Labicotes guttatus / P. superciliaris e Crax fasciolata). Para esta afirmação levamos em conta a possibilidade de "contaminação" quando trabalhando com material proveniente de Jardim Zoológico (Pilgrim \& Palma 1982) e sobretudo aquele que apresenta a interferência do homem (Hopkins 1939). O material encontrado no Zoológico de São Paulo em 2003 está em maioria condizente com a lista mundial de Price et al. (2003). Os achados de novos hospedeiros são coerentes, visto a afinidade entre os hospedeiros relatados. Note-se que os hospedeiros são do acervo vivo de zoológico e que os artrópodes recolhidos em cada exame individual foram guardados em recipientes separados para cada ave.

Cinco amostras não puderam ser identificadas especificamente (Myrsidea sp. em Cyanocorax cristatellus; Psittacobrosus sp. em Anodorhynchus hyacinthinus; Craspedorrhynchus sp. em Leucopternis polionota; Degeeriella sp. em Polyborus plancus; Oxylipeurus sp. em Aburria jacutinga) pois não há espécies descritas nestes respectivos gêneros sobre tais hospedeiros (Price et al. 2003) ou pela falta de concordância com as já descritas. As tentativas de identificação a partir da comparação com espécies descritas sobre hospedeiros mais próximos também não foram bem sucedidas. Com alguma exceção, é provável que estes espécimes sejam espécies novas para a ciência, devendo, portanto serem tratados em descrições criteriosas.

Myrsidea sp. encontrado em Cyanocorax cristatellus apresenta-se distinta morfologicamente das demais espécies de Myrsidea encontradas nos corvídeos, os espécimes agora encontrados poderiam se assemelhar a Myrsidea fallax Kéler, 1938 parasito de Cyanocorax cyanomelas (Vieillot, 1818) fato que não ocorreu. Por apenas ter recebido um espécime ninfa (III) não foi possível a identificação específica de Psittacobrosus sp. em Anodorhynchus hyacinthinus, provavelmente trata-se de Psittacobrosus hyacinthini Price \& Beer, 1968 espécie já encontrada no Brasil neste mesmo hospedeiro (Price \& Beer 1968; Price et al. 2003). As espécies do gênero Craspedorrhynchus tendem a ser espécieespecíficas com seus hospedeiros (Mey 2001), não há espécie deste gênero descrita sobre Leucopternis polionota (Price et al. 2003), sendo apenas reconhecida Craspedorrhynchus umbrosus (Carriker, 1903) no gênero Leucopternis (em L. semiplumbea Lawrence, 1861) (Mey 2001) descrita somente pelos machos, o fato de apenas encontrar fêmeas de Craspedorrhynchus sp. em Leucopternis polionota limita um diagnóstico preciso. A única espécie de Degeeriella encontrada em Polyborus plancus é D. pricei (Price et al. 
Tabela I. Espécies de Phthiraptera de aves silvestres do Zoológico de São Paulo, SP.

\begin{tabular}{|c|c|c|c|c|c|c|}
\hline \multirow{2}{*}{ Espécies de Phthiraptera } & \multicolumn{4}{|c|}{$\mathrm{N}^{\mathrm{o}}$ de malófagos } & \multirow{2}{*}{ Espécies de Hospedeiros } & \multirow{2}{*}{ Registros no Brasil } \\
\hline & Machos & Fêmeas & Ninfas & Total & & \\
\hline \multicolumn{7}{|l|}{$\begin{array}{l}\text { Subordem Amblycera } \\
\text { Laemobothriidae }\end{array}$} \\
\hline $\begin{array}{c}\text { Laemobothrion (L.) glutinans } \\
\text { Menoponidae }\end{array}$ & - & 1 & 1 & 2 & Coragyps atratus [Urubu-comum] & Nelson \& Price (1965) \\
\hline Amyrsidea $(C$.$) purpurascens { }^{\text {a }}$ & - & 2 & - & 2 & Penelope superciliaris [Jacu ${ }^{\mathrm{b}}$ & \\
\hline Colpocephalum heterosoma ${ }^{\text {a }}$ & 3 & 2 & - & 5 & Phoenicopterus chilensis [Flamingo] & \\
\hline Colpocephalum maculatum & 6 & 3 & - & 9 & Polyborus plancus [Caracara] & $\begin{array}{l}\text { Price \& Beer (1963a); } \\
\text { Valente et al. }(2001)\end{array}$ \\
\hline Colpocephalum mycteriae $^{\text {a }}$ & - & 4 & 3 & 7 & Jabiru mycteria[Tuiuiu $]^{\mathrm{b}}$ & \\
\hline Colpocephalum napiforme & - & 4 & - & 4 & Leucopternis lacernulatus [Gavião-pomba] ${ }^{\mathrm{b}}$ & Price \& Beer (1963a) \\
\hline Colpocephalum trichosum a & 5 & 4 & - & 9 & Vultur gryphus [Condor-dos-Andes] & \\
\hline Holomenopon brevithoracicum & 2 & 9 & 1 & 12 & Cygnus melanocoryphus [Cisne-do-pescoço-preto] & Brum et al.(2004) \\
\hline Holomenopon leucoxanthum & 4 & 5 & - & 9 & Dendrocygna bicolor [Marreca-caneleira] & Brum et al.(2004) \\
\hline Kurodaia $(C$.$) crassiceps a$ & 4 & 12 & 1 & 17 & Pulsatrix perspicillata [Mucurututu] & \\
\hline Menacanthus chaparensis a & 2 & 6 & - & 8 & Penelope superciliaris $[\mathrm{Jacu}]^{\mathrm{b}}$ & \\
\hline Myrsidea sp. $^{\text {a }}$ & 6 & 4 & - & 10 & Cyanocorax cristatellus [Gralha-do-campo] ${ }^{\mathrm{b}}$ & \\
\hline Psittacobrosus burmeisteri ${ }^{\text {a }}$ & 1 & 3 & 1 & 5 & Ara chloroptera [Arara-vermelha] & \\
\hline \multicolumn{7}{|l|}{$\begin{array}{c}\text { Subordem Ischnocera } \\
\text { Philopteridae }\end{array}$} \\
\hline Acidoproctus rostratus a & - & - & 1 & 1 & Dendrocygna bicolor & \\
\hline Ardeicola leucosoma & 7 & 6 & - & 13 & Jabiru mycteria & Kumar \& Tandan \\
\hline Brueelia addoloratoi & 2 & 2 & - & 4 & Turdus rufiventris [Sabiá-laranjeira] & $\begin{array}{l}(1971) \\
\text { Valim \& Serra-Freire }\end{array}$ \\
\hline Brueelia nitzschi ${ }^{\text {a }}$ & - & 4 & - & 4 & Cyanocorax cristatellus ${ }^{\mathrm{b}}$ & $(2003)$ \\
\hline Cotingacola dimorpha $a^{\text {a }}$ & 4 & 8 & - & 12 & Procnias nudicollis [Araponga] ${ }^{\mathrm{b}}$ & \\
\hline Craspedorrhynchus brevicapitis & $s$ a 3 & - & 1 & 4 & Rupornis magnirostris [Gavião-carijó] & \\
\hline Craspedorrhynchus sp. ${ }^{\text {a }}$ & - & 5 & - & 5 & Leucopternis polionota [Gavião-pomba ${ }^{\mathrm{b}}$ & \\
\hline Degeeriella elani ${ }^{\text {a }}$ & 8 & 12 & 2 & 28 & Elanus leucurus [Gavião-peneira] & \\
\hline Degeeriella sp. $^{\text {a }}$ & 1 & 1 & - & 2 & Polyborus plancus & \\
\hline \multirow[t]{2}{*}{ Labicotes guttatus a } & - & 1 & - & 1 & Penelope superciliaris ${ }^{\mathrm{b}}$ & \\
\hline & 8 & 11 & - & 19 & Crax fasciolata [Mutum-de-penacho] ${ }^{\mathrm{b}}$ & \\
\hline Oxylipeurus sp. ${ }^{\text {a }}$ & 2 & 1 & 1 & 4 & Aburria jacutinga [Jacutinga] ${ }^{\mathrm{b}}$ & \\
\hline Pectinopygus fregatiphagus a & 1 & - & - & 1 & Fregata magnificens [Fragata] & \\
\hline Strigiphilus heterurus a & - & 1 & - & 1 & Rhinoptynx clamator [Mocho-orelhudo] & \\
\hline \multirow[t]{3}{*}{ Struthiolipeurus struthionis } & 3 & 8 & - & 11 & Struthio camelus [Avestruz] & \\
\hline & & & & & & Oliveira et al.(2001); \\
\hline & 72 & 119 & 13 & 210 & & Ribeiro et al.(2004) \\
\hline
\end{tabular}

${ }^{a}$ Primeiro registro no Brasil $\quad{ }^{\mathrm{b}}$ Novo registro de hospedeiro

2003), após comparação morfológica entre os espécimes recolhidos no Zoológico de São Paulo, não foi possível confirmar a identidade específica destes malófagos. Não é conhecida nenhuma espécie de Oxylipeurus parasito do gênero Aburria (Price et al. 2003), sendo possível que estejamos tratando de uma nova espécie.

Pela primeira vez na literatura foram encontrados malófagos infestando Penelope superciliaris; Aburria jacutinga; Leucopternis lacernulata e Procnias nudicollis (Price et al. 2003); sinalizando a necessidade de aumentar a investigação para este grupo de insetos.

A partir de simples observação, como em Valim et al. (2004), pôde-se inferir a natureza hematófaga de Holomenopon brevithoracicum, aqui encontrada sobre o cisne-de-pescoçonegro, graças à presença de material vermelho escuro nos espécimes fixados em álcool e posterior confirmação de eritrócitos no trato digestório dos insetos, após observação com objetiva de imersão. Este fato ganha importância, uma vez que há espécies de menoponídeos (Cohen et al. 1991) atuando como hospedeiros intermediários no ciclo de filarídeos cardíacos de cisnes. Conseqüentemente, são necessários estudos mais aprofundados das espécies reconhecidamente hematófagas e suas possíveis implicações na transmissão de patógenos entre as aves.

Amostragens rotineiras de malófagos em aves apreendidas ou cativas poderão fornecer melhor entendimento sobre a diversidade da fauna dos Phthiraptera no Brasil, em função da dificuldade de captura de material na natureza. A coleta e estudo de material proveniente de aves de vida livre poderiam elucidar aspectos da ecologia e comportamento destes parasitos. 
Agradecimentos. A Ricardo L. Palma (Departamento de Entomologia, Te Papa Tongarewa Museum - Nova Zelândia) por críticas e sugestões nas identificações de novos hospedeiros. Aos revisores da Revista Brasileira de Entomologia pelas valiosas críticas e sugestões no formato original do manuscrito.

\section{REFERÊNCIAS}

Barker, S. C. 1994. Phylogeny and classification, origins, and evolution of host-louse associations of lice. International Journal of Parasitology 24: 1285-1293.

Brum, J. G. W.; M. A. Coimbra; A. P. Albano \& R. M. M. Paulsen. 2004. Malófagos de alguns Anseriformes silvestres no Rio Grande do Sul. Revista Brasileira de Parasitologia Veterinára 13: 331.

Clay, T. 1950. A preliminary survey of the distribution of the Mallophaga ("Feather lice") on the class Aves (Birds). Journal of the Bombay Natural History Society 49: 430-443.

Cohen, S.; M. T. Greenwood \& J. A. Fowler. 1991. The louse Trinoton anserinum (Amblycera: Phthiraptera), an intermediate host of Sarconema eurycerca (Filarioidea: Nematoda), a heartworm of swans. Medical and Veterinary Entomology 5: 101-110.

Freitas, M.F.L.; M. C. N. Botelho; A. S. Leite; V. S. Magalhães; A. E. Sobrinho; R. A. Oliveira; M. H. C. C. Oliveira \& J. B. Oliveira 2002. Ectoparasitos de aves silvestres mantidas em cativeiro no estado de Pernambuco, Brasil. Entomologia y Vectores 9: 2533.

Hopkins, G. H. E. 1939. Straggling in the Mallophaga. The Entomologist 72: 75-77.

Johnson, K. P. \& D. H. Clayon. 2003. The biology, ecology, and evolution of chewing lice. p. 449-476. In: R. D. Price; R. A. Hellenthal; R. L. Palma; K. P. Johnson \& D. H. Clayton. (eds.) The chewing lice: world checklist and biological overview. Illinois Natural History Survey Special Publication 24. X + 501pp.

Kumar, P. \& B. K. Tandan. 1971. The species of Ardeicola (Phthiraptera: Ischnocera) parasitic on the Ciconiidae. Bulletin of the British Museum (Natural History) - Entomology 26: $119-159$.

Mey, E. 2001. A new Craspedorrhynchus species (Phthiraptera: Ischnocera) from Austrália, with an annotated checklist of this chewing louse genus. Mitteilungen aus dem Museum für Naturkunde in Berlin (Deutsche entomologische Zeitschrift) 48: $117-132$.

Nelson, R. C. \& R. D. Price. 1965. The Laemobothrion (Mallophaga: Laemobothriidae) of the Falconiformes. Journal of Medical Entomology 2: 249-257.

Oliveira, H. H.; R. M. S. N. C. Guerra; M. P. Valim; T.D. Vilar; R. G. Rodrigues; L. H. S. Moreira; M. Amorim; G. S. Gazêta \& N. M.
Serra-Freire. 2001. Registro de Struthiolipeurus nandu (Mallophaga: Philopteridae) parasitando Struthio camelus (Aves: Struthioniformes) no Brasil. Jornal Brasileiro de Patologia 37: 64.

Palma, R. L. 1978. Slide-mounting of Lice: a detailed description of the Canada Balsan technique. The New Zealand Entomologist 6: $432-436$.

Pilgrim, R. L. C. \& R. L. Palma. 1982. A list of the chewing lice (Insecta: Mallophaga) from birds in New Zealand. National Museum of New Zealand (Miscellaneous Series): 1-32.

Price, R. D. \& J. R. Beer. 1963. Species of Colpocephalum (Mallophaga: Menoponidae) parasitic upon the Falconiformes. The Canadian Entomologist 95: 731-763, 1963a.

Price, R. D. \& J. R. Beer. 1968. The genus Psittacobrosus (Mallophaga: Menoponidae) of the Neotropical Psittaciformes. Annals of the Entomological Society of America 61: 261-276.

Price, R. D.; R. A. Hellenthal \& R. L. Palma. 2003. World checklist of chewing lice with host associations and keys to families and genera. p. 1-448. In: R. D. Price; R. A. Hellenthal; R. L. Palma; K. P. Johnson \& D. H. Clayton (eds.). The chewing lice: world checklist and biological overview. Illinois Natural History Survey Special Publication 24. X + 501pp.

Ribeiro, V. L. S.; M. M. Ribeiro; M. Dalmargo \& A. Bianco-Junior. 2004. Ocorrência de Struthiolipeurus struthionis (Gervais, 1844) (Phthiraptera: Philopteridae) em Struthio camelus Linnaeus, 1758 (Struthioniformes: Struthionidae) no Rio Grande do Sul, Brasil. Revista Brasileira de Parasitologia Veterinária 13: 332.

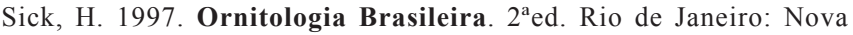
Fronteira. 862 p.

Silva, S. O.; H. H. Oliveira, R. H. F. \& N. M. Serra-Freire. 2004. Malófagos parasitas de aves campestres cativas do Zoológico Municipal Quinzinho de Barros, Sorocaba, Estado de São Paulo, Brasil. Entomologia y Vectores 11: 333-339.

Smith, V. S. 2001. Avian louse phylogeny (Phthiraptera: Ischnocera): a cladistic study based on morphology. Zoological Journal of the Linnean Society 132: $81-144$.

Valente, A. L. S.; R. M. M. Paulsen \& J. G. W. Brum. 2001. Colpocephalum maculatum Piaget, 1880 (Mallophaga: Menoponidae) em Gavião-Carijó (Rupornis magnirostris) e Caracará (Polyborus plancus) no Rio Grande do Sul. Arquivos da Faculdade de Veterinária. UFRGS 29: 147-148.

Valim, M. P. \& N. M. Serra-Freire. 2003. Novos registros de Amblycera e Ischnocera (Phthiraptera) em Turdus rufiventris (Vieillot, 1818) no município de Silva Jardim, Rio de Janeiro, Brasil. Lundiana 4: $155-156$.

Valim, M. P.; R. T. Serra-Freire; M. A. Fonseca \& N. M. Serra-Freire. 2004. Níveis de enzootia por ectoparasitos em amostras de rolinha [Columbina talpacoti (Temminck, 1810)] no Rio de Janeiro, Brasil. Entomologia y Vectores 11: 589-598. 